\title{
O Futebol no Centro de Atenção Psicossocial (CAPS): relato de experiência
}

\author{
Soccer at the Psychosocial Attention Center (CAPS): experience report
}

Fútbol en el Centro de Atención Psicosocial (CAPS): informe de experiencia

\author{
Murillo Henrique Santana ${ }^{I}$, Márcio Vinícius Brito CirqueiraII, Tadeu João Ribeiro Baptista ${ }^{\mathrm{III}}$
}

\begin{abstract}
Resumo
O tratamento de usuários de um Centro de Atenção Psicossocial (CAPS) pode ser feito de várias formas, sendo uma delas as práticas corporais, entre as quais se insere o futebol, pois este esporte, ainda que na dimensão do lazer, traz possibilidades educativas e de saúde. Este relato de experiência tem o objetivo de expor os CAPSs como campo de estágio e intervenção profissional da área de Educação Física (EF) e, mais ainda, de demonstrar como o futebol pode ser um ótimo recurso de tratamento para esses usuários. Foi feita pesquisa participante das aulas de futebol, por parte de um estagiário do curso de bacharelado em Educação Física, no segundo semestre de 2019, uma vez que ele observava e intervia no "Grupo de Futebol" planejando e discutindo, com o professor orientador do estágio e o professor do CAPS, as atividades que seriam aplicadas. $\mathrm{O}$ foco deste relato de experiência foi demonstrar como as práticas corporais possibilitam a interação profissional/ usuário, permitindo acompanhar as atividades como os jogos, além de demonstrar as contribuições para o grupo, como a redução do embotamento afetivo, a maior participação da atividade, uma melhoria nas habilidades técnicas. Identificou-se que o futebol pode trazer contribuições para o tratamento dos usuários em relação aos transtornos que apresentam, principalmente no que se refere à sociabilidade, volição e às potencialidades.
\end{abstract}

Palavras-chave: Educação física; Futebol; CAPS; Saúde mental

\begin{abstract}
The treatment of users of a Psychosocial Care Center (CAPS) can be done in several ways, one of which is bodily practices, including soccer, as this sport, even in the leisure dimension, brings educational and health related possibilities. This experience report aims to expose the CAPS as an internship and professional intervention field in the Physical Education (PE) area and, even more, to demonstrate how soccer can be a great treatment resource for these users. A Participant research of soccer classes was made by an intern of the bachelor's degree in Physical Education in the second half of 2019, since they observed and intervened in the "Soccer Group", planning and discussing with the internship's supervisory teacher and the CAPS teacher the activities that would be applied. The focus of this experience report was to demonstrate how bodily practices enable professional/user interaction, allowing to monitor activities such as games, as well as contributions to the group such as the reduction of affective dullness, greater participation in the activity, and improvement in technical skills. It was identified that soccer can help the treatment of users in relation to the disorders they present, especially regarding sociability, volition, and potential.
\end{abstract}

Keywords: Physical education; Soccer; CAPS; Mental health

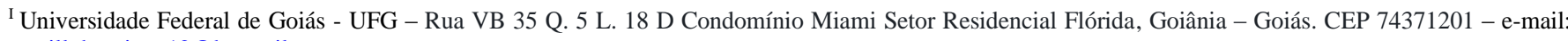
murillohenrique12@hotmail.com

II Secretaria Municipal de Saúde de Goiânia - e-mail: marcio muringa@yahoo.com.br

${ }^{\text {III }}$ Universidade Federal do Rio Grande do Norte - UFRN-e-mail: tadeujrbaptista@yahoo.com.br 


\section{Resumen}

El trato a los usuarios de un Centro de Atención Psicosocial (CAPS) se puede realizar de varias formas, una de las cuales son las prácticas corporales, entre las que se incluye el fútbol, porque este deporte, aunque en la dimensión del ocio, trae posibilidades de actividades educativas y de salud. Este relato de experiencia tiene como objetivo exponer los CAPS como campo de prácticas e intervención profesional en el ámbito de la Educación Física (EF) y, más aún, demostrar cómo el fútbol puede ser un gran recurso de tratamiento para los usuarios. La investigación participante de las clases de fútbol fue realizada por un pasante de la Licenciatura en Educación Física en el segundo semestre de 2019, ya que observó e intervino en el "Grupo de Fútbol" planificando y discutiendo con el docente supervisor de la pasantía y el docente de CAPS las actividades. que se aplicaría. El enfoque de este relato de experiencia fue demostrar cómo las prácticas corporales posibilitan la interacción profesional / usuario, permitiendo monitorear actividades como los juegos, así como contribuciones al grupo como la reducción del embotamiento afectivo, la mayor participación en la actividad y la mejora en habilidades técnicas. Se identificó que el fútbol puede contribuir al tratamiento de los usuarios en relación con los trastornos que presentan, especialmente en lo que respecta a la sociabilidad, la volición y el potencial.

Palabras clave: Educación Física; Fútbol; CAPS; Salud Mental

\section{Introdução}

Os Centros de Atenção Psicossocial (CAPSs) são unidades de saúde pública do Sistema Único de Saúde (SUS), que objetivam oferecer tratamento à população que sofre de transtornos psíquicos. São serviços substitutivos aos antigos hospitais psiquiátricos. A ideia é superar, tanto no tratamento, como na vida em sociedade, o a priori de que a pessoa com desordens mentais seja perigosa para o convívio social (ELIA et al., 2018). Os CAPSs foram estabelecidos pela Portaria 336 de 19 de fevereiro de 2002 e, conforme essa e outras portarias, institui-se a Rede de Atenção Psicossocial (RAPS) em todo o país. Os CAPSs são compostos por equipe multiprofissional, que atua de forma interdisciplinar, atendendo às pessoas com agravos do processo saúde-doença.

Os CAPSs podem dividir-se por especificidade da "saúde mental”. Esses serviços, independentes uns dos outros, embora façam parte da mesma RAPS, dividem-se em serviços que atendem as seguintes especificidades: transtornos mentais em menores de idade sem dependência química, transtornos mentais em menores de idade com dependência química, transtornos mentais em maiores de idade sem dependência química e transtornos mentais em maiores de idade com dependência química. No caso do CAPS $B^{1}$ o atendimento é exclusivo a maiores de idade sem dependência química. Não há exceções para atendimento de menores de idade em hipótese alguma, e as exceções de atendimento aos casos de dependência química em maiores de idade se restringe ao fato de a dependência ser uma comorbidade (não ser o transtorno mental principal). Cada caso é discutido individualmente a partir do momento em

${ }^{1}$ Usaremos a expressão “CAPS B” para garantir a anonimidade e confidencialidade da instituição. 
que a pessoa procura o CAPS para se informar e solicitar o acolhimento (serviço de triagem humanizado) pleiteando vaga para tratamento.

O CAPS B é um CAPS tipo $\mathrm{II}^{2}$, foi inaugurado em 2003, atende a região sudoeste do município, promovendo, à população com distúrbios mentais, tratamentos não convencionais, realizados por uma equipe multiprofissional, os quais não se limitam apenas ao uso de medicamentos psicotrópicos.

Geralmente, os CAPSs admitem novos usuários do serviço após triagem do caso, a qual, por orientação do Ministério da Saúde, é denominada "acolhimento".

\begin{abstract}
Quando a pessoa chega deverá ser acolhida e escutada em seu sofrimento. Esse acolhimento poderá ser de diversas formas, de acordo com a organização do serviço. O objetivo nesse primeiro contato é compreender a situação, de forma mais abrangente possível, da pessoa que procura o serviço e iniciar um vínculo terapêutico e de confiança com os profissionais que lá trabalham. (BRASIL, MINISTÉRIO DA SAÚDE, 2004, p. 15)
\end{abstract}

No caso do CAPS B, o acolhimento consiste em uma ou duas entrevistas (a segunda para aprofundamento do caso então acolhido) em que, mediante uma escuta qualificada, coleta-se o histórico de adoecimento mental da pessoa, o qual é posteriormente estudado em reuniões da equipe multidisciplinar, que acontecem semanalmente, e, só então, havendo concordância da equipe, é definido se o local de atendimento da pessoa é mesmo o CAPS B ou outra unidade de saúde, de acordo com a RAPS do município de Goiânia - outro CAPS mais próximo do endereço da pessoa, uma unidade básica de saúde, pronto-socorro ou o ambulatório municipal de psiquiatria (BRASIL, 2010) - ou mesmo instituições de ensino especial públicas ou conveniadas, clínicas populares, atendimento em programas de extensão de universidades.

Ter a opção de encaminhar pessoas com transtornos mentais para outras instituições e unidades de ensino ou de saúde, de acordo com o tipo de adoecimento ou mesmo de deficiência, depende da realidade de cada município, se os locais possuem essas instituições ou não, em maior ou menor quantidade e diversidade.

O transtorno mais comum entre os usuários do CAPS B é a esquizofrenia. Este transtorno se caracteriza pelas distorções do pensamento e alterações da sensopercepção, o que se expressa em delírios

\footnotetext{
${ }^{2}$ De acordo com a Portaria (MS) 336/2002, os serviços de atenção psicossocial (CAPSs) são divididos em tipos I, II e III. Os CAPSs I têm capacidade operacional para atendimento em municípios com população entre 20.000 e 70.000 habitantes e funcionam no período das $08 \mathrm{~h}$ às $18 \mathrm{~h}$, em dois turnos, durante os cinco dias úteis da semana. Os CAPSs II têm capacidade operacional para atendimento em municípios com população entre 70.000 e 200.000 habitantes, também funcionam das $8 \mathrm{~h}$ às $18 \mathrm{~h}$, em dois turnos, durante os cinco dias úteis da semana, podendo comportar um terceiro turno funcionando até às $21 \mathrm{~h}$. Os CAPSs II têm capacidade operacional para atendimento em municípios com população acima de 200.000 habitantes, e são serviços ambulatoriais de atenção contínua, durante 24 horas, diariamente, incluindo feriados e finais de semana.
} 
ou alucinações, pelo embotamento afetivo (dificuldade de expressar, vivenciar sentimentos e emoções), diminuindo a interação social (APA, 2013). As pessoas com esse transtorno carregam consigo um estigma que acarreta, muitas vezes, a exclusão social. Além disso, essa população tem uma ampliação de risco de mortalidade pelo uso crônico de medicamentos antipsicóticos, que podem acarretar doenças cardíacas (BEACH et al., 2018; ROSARIO et al., 2018) e outras doenças causadas por um estilo de vida sedentário, associado à má alimentação, ambos induzidos por sintomas como juízo crítico prejudicado e hipoatividade. E essa redução das atividades psicomotoras pode trazer outras dificuldades, como a lentificação das habilidades motoras finas e grossas, o que dificulta a execução de atividades cotidianas (BATTAGLIA et al., 2013).

Os usuários do serviço participam de dinâmicas terapêuticas em uma rotina prevista em agenda semanal de frequência de atividades, preferencialmente grupais, com a mesma finalidade chamada "projeto terapêutico singular", pensado de acordo com a individualidade de cada pessoa (BRASIL, 2012), buscando oferecer as melhores alternativas para as pessoas em tratamento. Além das atividades mais centradas na verbalização, como as rodas de conversa (divididas ou não entre sexos) e sessões de terapia individuais, os grupos terapêuticos podem envolver diversas práticas, como futebol, dança, boxe, música, pintura e atividades manuais.

Observando as vivências e grupos da área de Educação Física no CAPS B, um serviço que se fortalece por sua natureza interdisciplinar, vemos ali, claramente, um espaço de intervenção da Educação Física, em uma realidade em que as questões relativas à saúde mental das pessoas tomam uma proporção maior e convocam o SUS a assumir seu papel contributivo.

\footnotetext{
Por sua missão e configuração organizativa, o SUS é indiscutivelmente um campo de intervenção interprofissional. Pensar currículos integrados com os demais cursos da saúde, e, inclusive, fora da saúde, permite atender uma demanda primária do trabalho em saúde, qual seja, produzir cuidado de forma colaborativa. Na experiência da UNIFESP Campus Baixada Santista, por exemplo, os discentes de Educação Física, Fisioterapia, Nutrição, Psicologia, Serviço Social e Terapia Ocupacional compartilham eixos de formação que atravessam os seis semestres iniciais do curso, a saber: ser humano em sua dimensão biológica; o ser humano e sua inserção social; e a aproximação ao trabalho em saúde. Ao ampliar essa compreensão, entendo que o trabalho no ensino formal (licenciatura), bem como nos demais eixos de formação do bacharelado (esporte, e cultura e lazer), é necessariamente campo de intervenção interdisciplinar, e que também exige trabalho colaborativo de profissionais de distintas formações. (COSTA, 2019, p. 2)
}

Como todo profissional de nível superior lotado nos CAPSs de Goiânia (exceto médico), o professor de Educação Física é efetivamente responsável pela realização de acolhimento (inicial) de novos pacientes e de egressos (retorno feito pelo re-acolhimento) que estão restabelecendo o tratamento, bem como, pela proposição e planejamento de grupos e atividades terapêuticas gerais - visita domiciliar, 
atendimento a pacientes e familiares acerca do tratamento -, e específicas da própria área - atividades relacionadas à cultura corporal (dança, ginástica, lutas, esporte, jogo). Considerando que as práticas corporais podem contribuir com uma melhor consciência corporal, a relação com as emoções, com os quais, práticas esportivas (entre elas o futebol) podem contribuir na relação dos usuários com eles mesmos, outros usuários, os profissionais, a família e a comunidade.

O CAPS é um dos campos de Estágio oferecidos por uma instituição pública para a realização das disciplinas de Estágio Curricular Profissional III e IV do curso de bacharelado em Educação Física. O estágio tem como objetivo oferecer experiências de intervenção e aprimoramento da formação humana e profissional desenvolvidos ao longo do curso. Ademais, os discentes devem intervir e avaliar os resultados de sua ação. Portanto, o objetivo deste trabalho é apresentar as reflexões geradas a partir das experiências no campo de estágio, das aulas de futebol com um grupo de usuários da saúde mental.

\section{Metodologia}

Este trabalho é um relato de experiência, de abordagem qualitativa, baseado na pesquisa participante. Na primeira parte do estágio, realizada no primeiro semestre de 2019, no CAPS B, houve a orientação de os alunos de Educação Física observarem as atividades e, ao mesmo tempo, foram realizados estudos acerca das funções psíquicas e suas patologias mais comuns presentes em pacientes do CAPS B. Durante esse período, foram observados três grupos terapêuticos de práticas corporais: grupo de ritmos, de boxe e de futebol. Após a observação, os discentes propuseram intervenções, com uma atividade que tivesse objetivos para a realização, metodologia e avaliação dos resultados. Nesta instituição, o CAPS B, os alunos do estágio são acompanhados por um professor da Universidade e pelos profissionais do CAPS. Os professores acompanham as atividades de acordo com o número de grupos de estagiários, o que permite a presença do docente da universidade, em campo, pelo menos, uma vez por semana.

Inicialmente, havia quatro estagiários, todavia, no período de intervenção, apenas um acadêmico permaneceu no CAPS B. Por isso, o profissional de Educação Física do CAPS B sugeriu que o aluno assumisse a direção do grupo de futebol, o qual conta com aproximadamente doze usuários.

Após a realização das atividades, as observações realizadas no grupo eram relatadas oralmente e avaliadas por membros da equipe multiprofissional presentes no momento (mais comumente psicólogas e assistentes sociais), com a participação do estagiário e do professor orientador vinculado à universidade. Assim, profissionais e estagiário, reunidos no intervalo entre as atividades, discutiam sobre cada usuário: como ele agiu, qual era seu estado de humor, como estavam as funções psíquicas (melhoras, agudizações 
ou mesmo cronificação) e, principalmente, se foi identificada alguma alteração positiva ao longo do período de vinculação do usuário ao grupo de futebol. Ao final as observações e relatos mais relevantes (e até as ausências), as informações eram anotadas nos prontuários individuais de cada usuário participante do grupo.

\section{Reconhecimento de campo}

Para entender um pouco o contexto do atendimento nos Centros de Atenção Psicossocial, serão apresentadas, a seguir, algumas características da atuação no serviço de acordo com as propostas do Ministério da Saúde, assim como, algumas características específicas do CAPS B.

\subsection{Contextualizando o CAPS}

A definição de saúde mental da OMS (2004, p. 12), é “[...] um estado de bem-estar em que o indivíduo percebe suas próprias habilidades, podendo lidar com as tensões normais da vida, trabalhar produtiva e proveitosamente, sendo capaz de fazer uma contribuição para sua comunidade”. Esta é redefinida por Galderise et al. (2017, p. 408) como "[...] um estado dinâmico de equilíbrio interno que permite aos indivíduos usar suas habilidades em harmonia com os valores universais da sociedade; capacidade de reconhecer e expressar as próprias emoções, bem como ter empatia com o próximo" (tradução livre).

$\mathrm{O}$ atendimento do CAPS é dedicado às pessoas com

[...] qualquer síndrome caracterizada por distúrbios clinicamente significativos na cognição de um indivíduo, na regulação de emoções ou no comportamento que reflete uma disfunção nos processos psicológicos, biológicos ou de desenvolvimento subjacentes ao funcionamento mental" (APA, 2013, p. 20).

Sua criação veio da necessidade de substituir os modelos de tratamentos desumanos presentes nos hospícios e manicômios.

Os CAPS associam os tratamentos medicamentosos, indicados pelos psiquiatras, com uma gama ampla de terapias, a fim de proporcionar sua integração social e familiar, promovendo uma busca por autonomia, tanto do paciente, quanto da família (MILHOMEM; OLIVEIRA, 2007).

O CAPS desenvolve várias abordagens para reconstruir as perdas cognitivas, motoras e socioafetivas, capacitando os usuários a exercerem o direito à cidadania, quanto à família, moradia, 
trabalho e saúde (MINISTÉRIO DA JUSTIÇA, 2012, apud. SANCHES; VECCHIA, 2018). E, procura promover saúde, ou seja:

[...] ações transversais que possibilitem atender as necessidades sociais em saúde, com uma abordagem integral do processo saúde-doença, com foco no enfrentamento dos problemas de saúde baseado no reconhecimento dos determinantes sociais da saúde na sua produção; e dialogar com diversas áreas do setor sanitário, com setores do governo e com a sociedade com o objetivo de cuidado com a vida, e compondo redes de compromisso e corresponsabilidade (BRASIL, 2012, p. 29).

A promoção da saúde e o tratamento psiquiátrico se fazem por várias práticas, como as ações corporais/ atividades físicas, de lazer, por uma equipe multiprofissional atuando de forma interdisciplinar, com o objetivo de alargar a autonomia dos usuários - a capacidade de compreensão e atos críticos, tomando decisões sobre sua saúde e as relações sociais - advindo do acesso ao conhecimento (BRASIL, 2012).

Os transtornos mais comuns encontrados entre os usuários do CAPS são a esquizofrenia, o transtorno bipolar, a ansiedade, a depressão, o comprometimento dos comportamentos cognitivos (uma alteração nas funções de aprendizado como a percepção, a atenção e a memória), os fatores biológicos e mecânicos que influenciam o movimento corporal, inter-relacionados ao aprendizado, controle e desenvolvimento motor (GALLAHUE et al., 2013); e afetivo-social na relação com os sentimentos e emoções que o indivíduo tem com familiares, amigos, colegas de trabalho e a sociedade no geral (TAKEDA; STEFANELLI, 2006).

\subsection{CAPS B}

O Centro de Atenção Psicossocial B é um CAPS II e, por isso, funciona das $7 \mathrm{~h}$ às 19h, de segunda-feira a sexta-feira, formado por uma equipe com médico, psicólogo, enfermeiro, farmacêutica, terapeuta ocupacional, arteterapeuta e profissional de Educação Física.

Quando o acolhimento do usuário é feito e o caso é adequado ao CAPS (de moderado a grave), marca-se uma consulta com o médico psiquiatra e, após a discussão da equipe, a pessoa é direcionada ao cumprimento do projeto terapêutico singular - a rotina semanal de terapias e atividades no CAPS, que contribuam com o tratamento de seu quadro de adoecimento mental.

O espaço CAPES B está em uma chácara, alugada pela prefeitura municipal, e sua estrutura possui consultórios individuais, salas para grupos, espaço de convivência, salas para oficinas manuais e 
artísticas, refeitório (para oferecer refeições conforme o tempo de permanência do usuário no local), enfermaria e sanitários.

\section{Grupo de Futebol: intervenção, características dos usuários e resultados}

O grupo de futebol é coordenado por profissional de Educação Física concursado para a área de saúde mental do município de Goiânia, o qual desenvolve outras atividades além daquelas relacionadas diretamente à área de Educação Física - acolhimento, referência técnica de usuários do serviço (como elo entre esse usuário e sua família com o CAPS), visitas domiciliares, estudos de caso e planejamento do serviço junto à equipe multiprofissional. As atividades do grupo ocorrem em um campo de futebol society privado, cedido pelo proprietário. Para esta atividade, tem-se uma bola e 8 coletes ( 4 verdes e 4 azuis). Os usuários, antes das práticas corporais, aferem a pressão arterial e a glicemia na enfermaria.

Na oportunidade da coordenação dos jogos pelo professor e/ ou pelo estagiário, os usuários que participam do grupo são continuamente observados em suas falas e comportamentos todo o tempo antes, durante e depois das atividades propostas no dia - de forma discreta e sem demonstrar pretensões de observações ou investigações deliberadas, sem uso de registro específico durante a atividade. Destacase que, no CAPS B, o uso da medicação conforme prescrição médica é incentivado. Nos prontuários das pessoas atendidas pelo grupo de futebol, é possível acompanhar, pelas anotações de profissionais com diferentes formações, as melhoras dessas pessoas em sua sociabilidade, volição, entre outras.

Esse grupo ocorre desde outubro de 2014, em resposta a uma demanda crescente da parte de pacientes mais jovens pela prática do futebol, atividade também mencionada por Wachs (2007). Na história desse grupo, há relatos (descritos em prontuário) de pacientes e familiares (que eventualmente acompanham as atividades ou fazem comentários em reuniões privadas com o professor), os quais reportam as melhoras significativas no comportamento e nas funções psíquicas prejudicadas pelo adoecimento - principalmente no que se refere à volição, interação social e autoconfiança. Essas melhoras e sua estabilização não eram perceptíveis ou não se mantinham ao longo de um período de tempo mais longo até maior assiduidade no Grupo. Isso pôde ser observado mais recorrentemente em usuários mais presentes nas atividades dentro de um período de seis meses a um ano.

Durante o estágio, o grupo de futebol era composto, de forma geral, por homens, tendo bastante abertura para inclusão de novos participantes, inclusive mulheres. Ele era conduzido pelo professor de Educação Física durante o primeiro semestre, e foi assumido pelo estagiário durante sua intervenção no 
segundo semestre. Participavam, aproximadamente, 12 pessoas em dois jogos coletivos de futebol, com duração de 10 minutos cada, e intervalo de 5 minutos entre as partidas, realizadas às quintas-feiras, às $15 \mathrm{~h} 30$.

O estagiário foi orientado a distribuir os praticantes nas equipes com o maior equilíbrio possível entre os times em relação à defesa e ao ataque, e, se necessário, que fossem usadas estratégias para que esse equilíbrio fosse alcançado. Essas propostas variavam do uso da superioridade numérica de uma equipe em relação à outra ou alteração das regras, por exemplo: os jogadores de um time eram obrigados a passar a bola por um jogador com características mais reprimidas (embotamento de comportamento ou dificuldade técnica); um jogador mais acometido ser obrigado a dar no mínimo três toques na bola sem intervenção do adversário, entre outros.

Durante a atividade, os praticantes recebiam incentivos verbais para que tomassem iniciativas e atitudes relacionadas ao jogo: correr mais rápido, dominar a bola antes de chutar ou tocar, marcar o adversário, chutar ao gol antes de sofrer interceptação, entre tantas outras. Eles eram estimulados a comemorar; ou sendo uma situação de derrota, eles eram sacudidos a tomar iniciativas para mudar a situação como marcar o adversário, tocar a bola e atacar mais. Os mais acometidos eram os mais instigados.

O foco central era promover a possibilidade de praticar o futebol, incentivar a inclusão dos participantes nessa prática, já que, em outro contexto, devido ao estigma que as pessoas carregam com a esquizofrenia, elas seriam excluídas. As avaliações eram feitas após a realização do grupo em um diálogo com a equipe multiprofissional, e, após a análise em conjunto com a equipe, a conclusão era descrita no prontuário.

O futebol, em geral, tinha desfechos bastantes positivos para os usuários. Um exemplo é o usuário $\mathrm{A}^{3}$, que tinha muita dificuldade de realizar as atividades do grupo devido ao seu alto grau de embotamento afetivo e sua dificuldade técnica. Ao longo do tempo, pôde-se observar ganhos, sobretudo, nos aspectos psicológicos. Evidenciou-se a mudança de um comportamento mais acanhado e desmotivado e uma postura reprimida, para um comportamento com maior empenho e vontade, uma fisionomia alegre nas atividades do CAPS e, mesmo tendo dificuldades técnicas, sua disposição era alta para jogar futebol, aumentando seu protagonismo. Esse tipo de melhora também é demonstrado por Abib et al (2010).

Outro caso interessante foi o do usuário B, que chegou recentemente ao CAPS muito acometido pelos medicamentos, com embotamento afetivo intenso. Era possível observar alguma habilidade técnica

${ }^{3}$ Foram usadas letras para não divulgar os nomes dos pacientes. 
com a bola, mesmo com sua motricidade comprometida. Do dia em que ele iniciou o tratamento até o último dia de estágio, tornaram-se evidentes a fisionomia mais alegre, a vontade de ir ao CAPS, participar da atividade, as comemorações durante as situações de jogo, inclusive em seus vários gols.

Battaglia et al. (2013) fizeram uma pesquisa com 4 indivíduos diagnosticados com esquizofrenia, submetidos a 12 semanas de treinamento de futebol. Eles fizeram uma avaliação subjetiva da qualidade de vida e uma de performance do esporte, no pré e no pós-treinamento. Compararam-se os resultados com um grupo controle de 4 indivíduos, diagnosticados com o mesmo distúrbio, e apresentaram-se melhoras consideráveis nos testes físicos e no teste subjetivo de qualidade de vida do grupo treinado, sendo este o principal alvo do tratamento de indivíduos com esquizofrenia. Os pesquisadores concluíram que o futebol é útil na melhoria da "saúde psicofísica" de pessoas com esquizofrenia, como redução de sintomas psicóticos, redução do uso de medicamentos, aumento da interação social e evolução da movimentação corporal, sendo uma terapia alternativa junto à terapia farmacológica.

Aumentar as funções psicomotoras contribui na melhoria da saúde mental de pessoas em quadros psicóticos, pois, devido às atividades motoras reduzidas, dificultam-se as relações sociais e atividades cotidianas (MORRENS; HULSTIJN; SABBE, 2007). Soundy et al. (2015) analisaram o impacto na perda de peso, nos sintomas psicóticos e em outros aspectos da saúde de indivíduos com esquizofrenia inseridos em programas de esportes. Segundo eles, o esporte tem o potencial de melhorar a qualidade de vida de uma pessoa, fornecendo uma atividade significativa que leva à conquista, sucesso e satisfação.

\section{Considerações finais}

Nas experiências dos dois momentos do estágio (o primeiro em que predominou a observação e estudo da área de saúde mental e o segundo momento, de maior diretividade por parte do estagiário remanescente do grupo) foi possível observar que, mesmo sem muitos recursos financeiros, é exequível fazer um trabalho que transforme a vida de pessoas. Foram notáveis os benefícios que a Educação Física pode proporcionar, através de práticas corporais para pessoas que, até então, estavam excluídas dessas atividades.

O estigma das pessoas com distúrbios mentais acaba por excluí-las socialmente. Observou-se no CAPS uma batalha contra esse estereótipo, com atitudes que permitem aos usuários a realização de atividades que tragam algum tipo de reflexão sobre seu valor como ser humano para a sociedade.

O estágio no CAPS B foi importante, também, no processo de formação do aluno, fazendo-o ainda mais humano, diminuindo seus preconceitos e abrindo seu olhar aos problemas dos usuários, além da 
reflexão sobre como ajudá-los. Despertou a curiosidade para o alcance terapêutico de natureza mental/ emocional da prática corporal, pouco explorada durante a graduação.

O futebol, como prática corporal coletiva, demonstra potencial no tratamento de pessoas com transtorno mental. O foco deste relato de experiência foi demonstrar como as práticas corporais, possibilitam a interação profissional/ usuário, permitindo acompanhar as atividades, por um lado, em meio a simples situações como comemorar um gol e, por outro, facilitar ao usuário a percepção de suas potencialidades.

A experiências observadas no estágio, e documentadas em prontuários dos pacientes, acerca das práticas corporais no CAPS B, são sugestivas e carecem de mais investigações, com metodologias e análises diversas, para se vislumbrar com maior clareza os alcances e limites das práticas corporais integradas ao trato de funções psíquicas das pessoas adoecidas. Enfim, o CAPS é potente para formação dos alunos de Educação Física. Apesar dos desafios enfrentados, o trabalho envolve grandes potencialidades no âmbito da promoção de saúde.

\section{Referências}

ABIB, L. T. et al. Práticas corporais em cena na saúde mental: potencialidades de uma oficina de futebol em um Centro de Atenção Psicossocial de Porto Alegre. Pensar a Prática, v. 13, n. 2, p. 1-15, 2010.

AMERICAN PSYCHIATRIC ASSOCIATION (APA). Diagnostic and statistical Manual of Mental Disorders. 5. ed. Arlington, VA: APA, 2013.

BATTAGLIA, G. et al. Soccer practice as an add-on treatment in the management of individuals with a diagnosis of schizophrenia. Neuropsychiatric Disease and Treatment, v. 9, p. 595-603, 2013.

BEACH, S. et al. QT Prolongation, Torsades de Pointes, and Psychotropic Medications: A 5-Year Update. Psychosomatics, v. 59, n. 2, p. 105-122, Mar.-Apr. 2018.

BRASIL. Ministério da Saúde (MS), Secretaria de Atenção à Saúde, Núcleo Técnico da Política Nacional de Humanização. Acolhimento nas práticas de produção de saúde. 2. ed. 5. reimp. Brasília, DF: Ministério da Saúde, 2010.

BRASIL. Ministério da Saúde (MS). Secretaria-Executiva. Secretaria de Vigilância em Saúde. Glossário temático: promoção da saúde. Brasília: Ministério da Saúde, 2012.

COSTA, Filipe Ferreira. Novas diretrizes curriculares para os cursos de graduação em Educação Física: oportunidades de aproximações com o SUS? Rev Bras Ati. Fis Saúde, 24, p.1-4, 2019.

ELIA, D. et al. Relatório 30 Anos de SUS: Que SUS para 2030? Brasília: Ministério da Saúde, 2018. 
GALDERISI, S. et al. A proposed new definition of mental health. Psychiatria Polska, v. 51, n. 3, p. 407-411, 2017.

GALlAHUE, D. L. et al. Compreendendo o Desenvolvimento Motor. 7. ed. Porto Alegre: AMGH, 2013.

MIKKELSEN, K. et al. Exercise and mental health. Maturitas, v. 106, p. 48-56, 2017.

MILHOMEM, M. A. G. C.; OLIVEIRA, Alice G. B. de. O trabalho em equipe nos Centros de Atenção Psicossocial-CAPS. Cogitare Enfermagem, v. 12, n. 1, p. 101-108, 2007.

MORRENS, M.; HULSTIJN, W.; SABBE, B. Psychomotor slowing in schizophrenia. Schizophrenia Bulletin, v. 33, n. 4, p. 1038-1053, 2007.

ROSARIO, L. H. Martín et al. Antipsychotics and cardiovascular risk: A case/non-case study. Psychiatry Research, v. 270, p. 341-347, Dec. 2018.

SANCHES, L. R.; VECCHIA, M. D. Reabilitação Psicossocial E Reinserção Social De Usuários De Drogas: Revisão Da Literatura. Psicologia \& Sociedade, v. 30, n. 0, p. 1-10, 2018.

SOUNDY, A. et al. Investigating the benefits of sport participation for individuals with schizophrenia: A systematic review. Psychiatria Danubina, v. 27, n. 1, p. 2-13, 2015.

TAKEDA, O. H.; STEFANELLI, M. C. Atividade física, saúde mental e reabilitação psicossocial. Revista Mineira de Enfermagem, v. 10, n. 11, p. 171-175, 2006.

WACHS, F. Educação física e o campo da saúde mental: uma reflexão introdutória. In. Alex Branco Fraga e Felipe Wachs (Org). Educação Física e Saúde Coletiva: políticas de formação e perspectivas de intervenção. 2. ed. UFRGS. Porto Alegre - RS. 2007, p. 87-98.

WORLD HEALTH ORGANIZATION (WHO). Promoting mental health: concepts, emerging evidence, practice. Geneva: World Health Organization, 2004.

\section{Como citar este artigo}

SANTANA, M. H.; CIRQUEIRA, M. V. B.; BAPTISTA, T. J. R. O Futebol no Centro de Atenção Psicossocial (CAPS): relato de experiência. Revista Kinesis, Santa Maria, v. 39, p.0113, 2021.

* O presente trabalho não contou com apoio financeiro de nenhuma natureza para sua realização. 